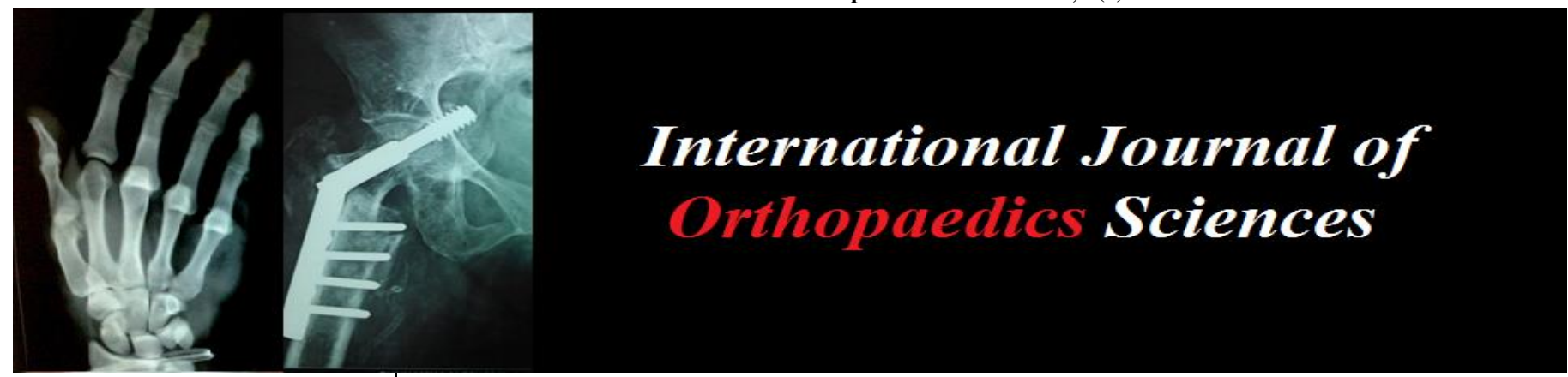

ISSN: $2395-1958$

IJOS 2018; 4(2): 88-90

(C) 2018 IJOS

www.orthopaper.com

Received: 13-02-2018

Accepted: 14-03-2018

Dr. Dasarath Kisan

Associate Professor, Department of Orthopaedics, Hi-tech Medical College and Hospital, Rourkela Odisha, India

Saswat Samant

Senior Resident, Department of Orthopaedics, Kalinga Institute of Medical Science,

Bhubaneswar, Odisha, India
Correspondence

Saswat Samant

Senior Resident, Department of Orthopaedics, Kalinga Institute of Medical Science,

Bhubaneswar, Odisha, India

\section{A comparison of closed intramedullary nailing with open intramedullary nailing in femoral shaft fractures of adults}

\section{Dr. Dasarath Kisan and Dr. Saswat Samant}

DOI: https://doi.org/10.22271/ortho.2018.v4.i2b.13

\section{Abstract}

The overall incidence of femoral shaft fractures as well as their burden on society continues to be significant. The purpose of our study was to assess the results of intramedullary nailing of femoral shaft fractures by both open and closed methods. We made a retrospective study of 64 femoral shaft fractures that had been operated between 2012 to 2016 . The average healing time as well as the surgical operative duration was higher in open intramedullary nailing in comparison to the closed group. We believe that a more comprehensive study may be required for validation of these results.

Keywords: Femur shaft, intramedullary nailing, open nailing, closed nailing

\section{Introduction}

Femoral shaft fractures continue to be a heavy burden on the healthcare resources of any nation. Although the overall incidence of femoral shaft fractures continues to be less in comparison to other long bone fractures (American population-13/100000 [1], European population-10 to $13 / 100000^{[2-7]}$ the strain on resources as well as the amount of DALY is significant. This is especially true in a resource scarce developing nation with an enormous population like India. Since the time Kuntschner introduced the concept of intramedullary nailing, there have been tremendous advances enabling us to shift from open methods to closed techniques using a remote entry site at the proximal femur. In parallel, we have witnessed the improvements in nail design as well as instrumentation. Unfortunately, the health scenario in developing nations like India is handicapped with gross constraints like unequipped peripheral hospitals as well as less expertise among ancillary support staff. These general factors augment our requirement for not disposing off the older method of open fixation with intramedullary rodding for femoral nails. In complex fracture geometry as well as commented fractures, often reduction poses a problem when we go for closed nailing. An open approach ultimately helps in such a scenario. The purpose of our study was to assess the results of intramedullary nailing of femoral shaft fractures by both open and closed methods.

\section{Materials and Methods}

This was a retrospective study of 64 femoral shaft fractures that had been operated between 2012 to 2016. Patients above the age group of 18 years were included in our study. The cut of age limit had been chosen to exclude all paediatric cases. No upper age limit was fixed. 46 of the cases had been operated with closed intramedullary nailing while 28 of the cases had been managed with open intramedullary nailing. All the cases included in the study had stable general condition at the time of surgery. We excluded all femoral fractures which had been managed by traditional healers for prolonged periods of time. Cases that had follow up of at least 52 weeks were included in the study. All cases of multiply fractures, polytrauma were excluded from the study as we attempted to evaluate only solitary femoral shaft fractures. Also excluded were cases which had insufficient documentation of individual case data as our study was a purely retrospective one. The standard protocol that had been employed by the surgeon for the post-operative rehabilitation included quadriceps drill along with hip and knee joint mobilization as soon as pain permitted. The negative suction drains were removed by $2 \mathrm{nd}$ 
postoperative day and stitches/staples were removed between 12-14th postoperative day. Standard antibiotic protocol had been followed in each case. Initial mobilization was with the help of a standard walking frame. Weight bearing was individualised as per fracture pattern and as tolerated. The patients had been followed up at 2, 4, 12, 24, 36 and 52 weeks postoperatively and the data that had been collected was used to assess the result of the surgeries.

\section{Results}

The ratio of male to female patients was $7: 1$ and the mechanism of injury reported was high energy road traffic accidents, low energy falls the road traffic accidents included motor vehicle crashes, motorcycle accidents as well as pedestrians struck by vehicles. All the cases were classified from their radiographs as per Winquist and Hansen ${ }^{[11,12]}$ as well as anatomically as being proximal, middle or distal third fractures of the shaft of femur. Majority of the cases were fractures in the middle $1 / 3 \mathrm{rd}$ of the shaft of femur
Table 1: Distribution of the fractures as per the anatomical location

\begin{tabular}{|c|c|c|}
\hline Proximal third & Middle third & Distal third \\
\hline 12 & 42 & 10 \\
\hline
\end{tabular}

We did not encounter any case of nerve palsy or refractures either in the open nailing group or in the closed nailing group. The rate of (superficial+deep) infections was slightly greater in fractures treated by open nailing (21.42\% VS $17.39 \%)$. The percentage of cases progressing towards union was almost similar at $14 \%$ and $13 \%$ respectively. We found the rate of malalignment being slightly lower in cases managed by open nailing $(7.14 \%$ vs $10.89 \%)$. The rate of knee / hip pain recorded on post op visits were around $14 \%$ and $7 \%$ in open nailing. This was $13 \%$ and $6.5 \%$ in closed cases. In $7.14 \%$ (2) of the open nailing cases we found the interlocking bolts being broken. In $10.89 \%$ (5) cases of closed nailing did we find any evidence of nail breakage or screw breakage

Table 2: Complications in intramedullary nailing

\begin{tabular}{|c|c|c|c|c|}
\hline & \multicolumn{2}{|c|}{ Open nailing } & \multicolumn{2}{c|}{ Closed nailing } \\
\hline & No. of cases & \% & No. of cases & \% \\
\hline Infection & 6 & $21.42 \%$ & 8 & 17.39 \\
\hline Non-union & 4 & 14.28 & 6 & 13.04 \\
\hline Malalignment (angular/rotational) & 2 & 7.14 & 5 & 10.89 \\
\hline Pain(knee/hip) & $4 / 1$ & $14 / 7$ & $6 / 3$ & $13.04 / 6.5$ \\
\hline Broken nail or interlocking screws & 2 & 7.14 & 5 & 10.89 \\
\hline Nerve injury & 0 & 0 & 0 & 0 \\
\hline Refracture & 0 & 0 & 0 & 0 \\
\hline
\end{tabular}

The average duration required for union ranged from 124 days to 236 days in patients who didn't go into non-union. The average healing time was lower for closed intramedullary nailing. Dynamization was required in 5 of the cases which underwent open nailing and 11 of the cases which underwent closed nailing. Mean hospital stay was 11 days for the fractures treated with open nailing and 9 days for fractures treated with closed nailing. (Overall duration range being 522 days)

Table 3: Other parameters

\begin{tabular}{|c|c|c|c|c|}
\hline & \multicolumn{2}{|c|}{ Open nailing } & \multicolumn{2}{c|}{ Closed nailing } \\
\hline & No. & $\mathbf{\%}$ & No. & $\%$ \\
\hline Dynamization required & 5 & 17.85 & 11 & 23.91 \\
\hline Average duration for union & $\begin{array}{c}186 \\
\text { days }\end{array}$ & N.A & $\begin{array}{c}146 \\
\text { days }\end{array}$ & N.A \\
\hline Mean hospital stay duration & 11 & N.A & 9 & N.A \\
\hline $\begin{array}{c}\text { Mean duration of surgery } \\
\text { (mins) }\end{array}$ & \multicolumn{2}{|c|}{84 mins } & \multicolumn{2}{|c}{66 mins } \\
\hline
\end{tabular}

\section{Discussion}

In our review of literature we found a few other comparisons in results of open vs closed intramedullary nailing. But the open nailings in these studies entailed absolutely no use of intraoperative fluoroscopy. The purpose being to evaluate the the results of open nailing in situations which did not have facilities of intraoperative fluoroscopy especially in remote and peripheral regions of developing countries like India. On the other hand we were studying the results of open nailing with the use of fluoroscopy as our aim was to study the results of open nailing vs closed nailing rather the effects of fluoroscopy acting as an aiding tool.

One of the drawbacks of our study was the very low number
Of cases, i. e only 28 cases of open intramedullary nailing. Other than this our study was neither prospective nor did it entail matching. The fracture pattern as per fracture geometry was grossly dissimilar. Apart from this the reason for undertaking an open or closed interlocking can also be the reason for the dissimilar post op results.

The average healing time was slightly higher in open intramedullary nailing in comparison to the closed group. These results are in line with what was shown by Pati et al ${ }^{[13]}$ and Meena et al ${ }^{[14]}$. The earlier healing time with close nailing is often attributed to the non-disturbance of the fracture haematoma and biology.

The duration of surgery was also more predictable and less despite the fact that most of the cases which were selected for open nailing ofen were old non-unions which required opening of the intramedullary canals as well as bone grafting. Due to direct visualization, the better anatomical reduction is more commonly possible. This leads to rarer results in rotational and angular malalignment.

\section{Conclusion}

Although it is agreed that in open nailing the surgical skin scar, the increased blood loss, the loss of fracture biology, the increased infection rate and decreased rate of union are genuine disadvantages of the procedures yet the convenience for a less experienced orthopaedic surgeon, ease in obtaining anatomical reduction as well as easier opening of medullary opening of sclerotic bones and the more predictable surgical time even in more complex comminuted fractures make this procedure quite attractive. We believe that a more comprehensive prospective study is required for comprehensive validation of these results. 


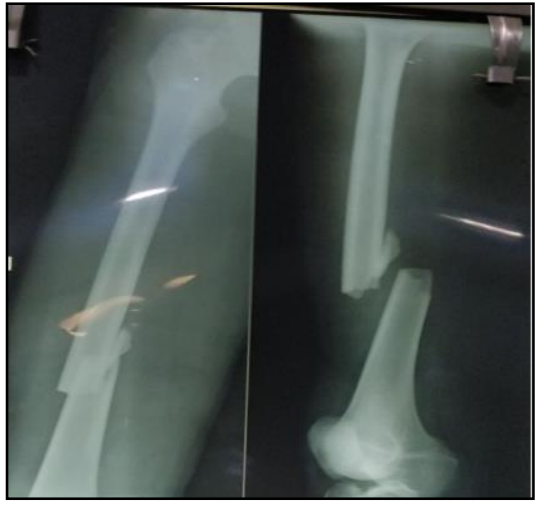

Fig 1: Preoperative radiograph

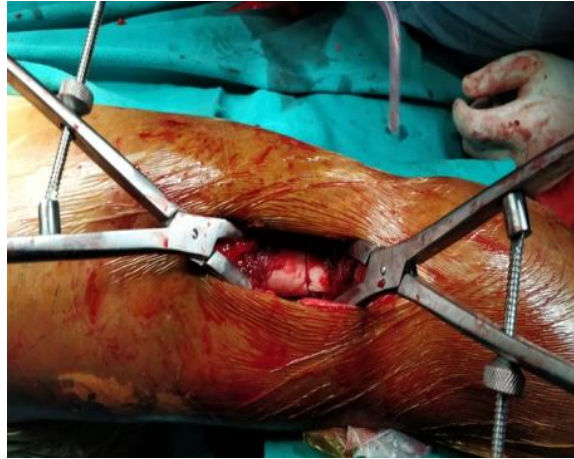

Fig 2: Intra operative images of open intramedullary nailing

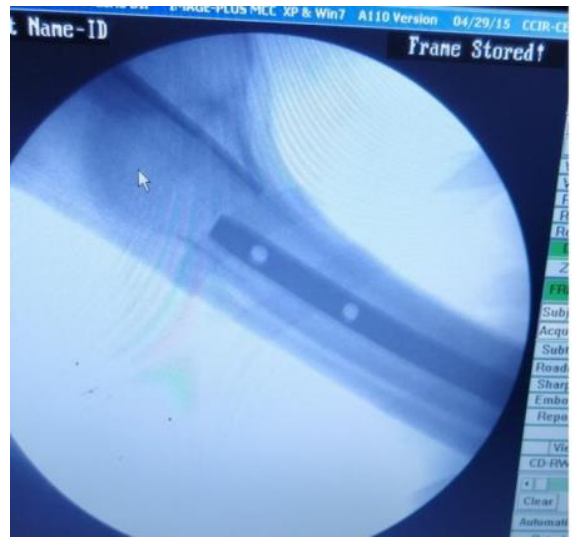

Fig 3: Intraoperative fluoroscopy image

\section{References}

1. Fakhry SM, Rutledge R, Dahners LE, Kessler D. Incidence, management and outcome of femoral shaft fractures: a statewide population-based analysis of 2805 adult patients in a rural state. J Trauma. 1994; 37:255261.

2. Weiss RJ, Montgomery SM, Al Dabbagh Z, Jansson KA. National data of 6409 Swedish inpatients with femoral shaft fractures: stable incidence between 1998 and 2004. Injury. 2009; 40:304-308.

3. Court-Brown CM, Caesar B. Epidemiology of adult fractures: a review. Injury. 2006; 37:691-697.

4. Salminen ST, Pihlajamaki HK, Avikainen VJ, Bostman OM. Population based epidemiologic and morphologic study of femoral shaft fractures. Clin Orthop Relat Res. 2000; 372:241Y249.

5. Court-Brown CM, Rimmer S, Prakash U, McQueen MM. The epidemiology of open long bone fractures. Injury. 1998; 29:529-534.

6. Loder RT, O’Donnell PW, Feinberg JR. Epidemiology and Mechanisms of Femur Fractures in Children. $\mathrm{J}$
Pediatr Orthop. 2006; 26:561-566.

7. Giannoudis PV, Giannoudis M, Stavlas P. Damage control orthopaedics: lessons learned. Injury. 2009; 40S4:47-52.

8. Kuntscher G. Die Marknagelung von Knochenbruchen. Arch Klin Chir. 1940; 200(443).

9. Kuntscher G. Further progress in the area of medullary nailing. Langenbecks Arch

Chir. 1966; 316:224-231.

10. Kuntscher G. Intramedullary nailing of comminuted fractures. Langenbecks Arch Chir. 1968; 322:1063-1069.

11. Bosse MJ, MacKenzie EJ, Riemer BL. Adult respiratory distress syndrome, pneumonia, and mortality following thoracic injury and a femoral fracture treated either with intramedullary nailing with reaming or with a plate. A comparative study. J Bone Joint Surg Am. 1997; 79A:799-809.

12. Bouchard JA, Barei D, Cayer D. Outcome of femoral shaft fractures in the elderly. Clin Orthop Relat Res. 1996:105-109.

13. Pati BN, Bansal VP, Krishna LG, Ahmed A, Garg S. Interlocking nail of femur. A review of 90 classes. Ind J Orthop. 2001; 35(1):49-51.

14. Meena R, Kundnani V, Hussain Z. Fracture of the shaft of the femur: Close vs open interlocking nailing. Indian Journal of Orthopaedics. 2006; 40(4):243. 